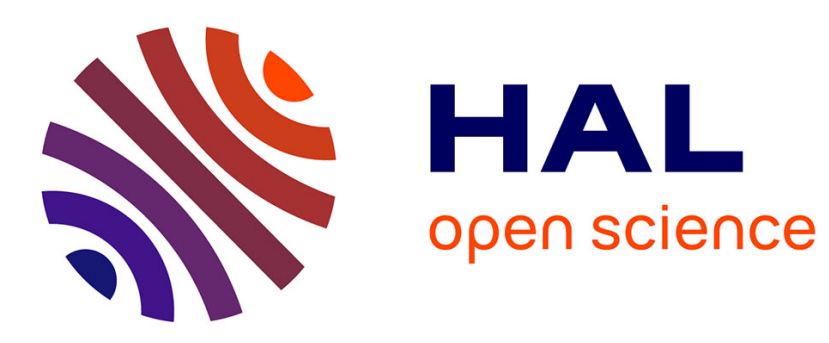

\title{
Orientia tsutsugamushi, the causative agent of scrub typhus, induces an inflammatory program in human macrophages.
}

Wiwit Tantibhedhyangkul, Amira Ben Amara, Julien Textoris, Laurent Gorvel, Eric Ghigo, Christian Capo, Jean-Louis Mege

\section{To cite this version:}

Wiwit Tantibhedhyangkul, Amira Ben Amara, Julien Textoris, Laurent Gorvel, Eric Ghigo, et al.. Orientia tsutsugamushi, the causative agent of scrub typhus, induces an inflammatory program in human macrophages.. Microbial Pathogenesis, 2012, epub ahead of print. 10.1016/j.micpath.2012.10.001. inserm-00749203

\section{HAL Id: inserm-00749203 https://www.hal.inserm.fr/inserm-00749203}

Submitted on 7 Nov 2012

HAL is a multi-disciplinary open access archive for the deposit and dissemination of scientific research documents, whether they are published or not. The documents may come from teaching and research institutions in France or abroad, or from public or private research centers.
L'archive ouverte pluridisciplinaire HAL, est destinée au dépôt et à la diffusion de documents scientifiques de niveau recherche, publiés ou non, émanant des établissements d'enseignement et de recherche français ou étrangers, des laboratoires publics ou privés. 


\title{
Orientia tsutsugamushi, the causative agent of scrub typhus, induces an inflammatory program in human macrophages
}

\author{
Wiwit Tantibhedhyangkul, ${ }^{1}$ Amira Ben Amara, Julien Textoris, \\ Laurent Gorvel, Eric Ghigo, Christian Capo, Jean-Louis Mege
}

\begin{abstract}
Aix-Marseille Université, URMITE, UMR CNRS 7278, IRD 198, Inserm 1095, 13005
Marseille, France

${ }^{1}$ Present address: Department of Immunology, Faculty of Medicine Siriraj Hospital, Mahidol University, Bangkok, Thailand.
\end{abstract}

Corresponding author: Jean-Louis Mege, URMITE, 27 Bld. Jean Moulin 13385 Marseille Cedex 5, France. Tel: +33 (0) 4913249 70, Fax: +33 (0) 4913877 72. E-mail: jeanlouis.mege@univ-amu.fr

E-mail WT: wiwit167@yahoo.co.th. E-mail ABA: bnounouaa@yahoo.fr. E-mail JT: julien.textoris@ap-hm.fr. E-mail EG: eric.ghigo@univ-amu.fr. E-mail CC: christian.capo@univ-amu.fr. E-mail JLM: jean-louis.mege@univ-amu.fr

Abbreviations: fetal bovine serum, FBS; Fold Change, FC; Gene Ontology, GO; heat-killed, HK; interferon, IFN; interferon-stimulated gene, ISG; oligomerization domain, NOD; quantitative real-time PCR, qPCR; TdT-mediated dUTP nick-end labeling, TUNEL; Tumor Necrosis Factor, TNF. 
Keywords: Orientia tsutsugamushi; macrophages; type I interferon; inflammatory cytokines; chemokines; apoptosis. 


\section{Introduction}

Orientia tsutsugamushi is the causative agent of scrub typhus, a tropical infectious disease endemic to the Asia-Pacific region, and is responsible for one million new infections each year. O. tsutsugamushi is transmitted to humans by the bite of larval trombiculid mites, and an estimated one billion people are at risk of contracting the infection [1]. Scrub typhus is associated with fever, rash and eschar. The clinical forms of scrub typhus include pneumonitis and meningitis, which may lead to multiorgan failure and death [2]. To date, no effective preventive strategy can provide long-lasting immunity, despite attempts to develop a prophylactic vaccine for scrub typhus [3].

O. tsutsugamushi belongs to a genus that has been separated from the genus Rickettsia on the basis of genetic and phenotypic differences, which include the absence of peptidoglycan and lipopolysaccharide in its cell wall [4]. The genomes of the Boryong and Ikeda strains have recently been sequenced $[5,6]$. O. tsutsugamushi is considered to be an intracellular pathogen that primarily infects endothelial cells both in vitro [7] and in vivo [8]. In addition, it appears that the interaction of O. tsutsugamushi with myeloid cells plays a pivotal role in $O$. tsutsugamushi infection [9]. In mice, O. tsutsugamushi infects and multiplies in peritoneal macrophages $[10,11]$, leading to the death of infected animals. However, mice are able to resist to infection if pretreated with a macrophage-activating agents such as BCG [12]. In humans, tissues autopsied from patients showing clinical symptoms of scrub typhus revealed $O$. tsutsugamushi in macrophages from the liver and the spleen [8]. We showed that $O$. tsutsugamushi replicates in human monocytes isolated from healthy donors and that infection alters the expression of a large number of host genes [13]. These findings were strenghtened by recently published data demonstrating the tropism of $O$. tsutsugamushi for monocytes and dendritic cells within eschars [14]. 
The mechanisms that govern local and systemic inflammation in scrub typhus have

only been partially elucidated. In patients with scrub typhus, the circulating levels of inflammatory cytokines and chemokines are elevated [15-17]. Similarly, the susceptibility of mice to $O$. tsutsugamushi infection is associated with the overproduction of cytokines and chemokines $[9,18]$. The expression of genes associated with the inflammatory response and of the interferon-stimulated genes (ISGs) is upregulated in human monocytes stimulated with O. tsutsugamushi. In addition, several features of M1 polarization are observed in circulating leukocytes from patients with scrub typhus [13]. As the half-life of monocytes in the blood is limited, the infection of tissue macrophages by $O$. tsutsugamushi may be independent of circulating cells.

In this study, we demonstrate that $O$. tsutsugamushi is able to replicate within human monocyte-derived macrophages and that it induces a robust transcriptional response in ISGs, apoptosis-related genes and genes encoding inflammatory mediators. Infection with $O$. tsutsugamushi also induces the release of interferon (IFN)- $\beta$ and inflammatory cytokines. Although this exacerbated inflammatory response is not sufficient to eradicate $O$. tsutsugamushi, it may account for the inflammatory complications of scrub typhus.

\section{Materials and Methods}

\subsection{Bacteria and cell culture}

O. tsutsugamushi, strain Kato (CSUR R163), was propagated and titered in L929 cells as described [13]. In brief, infected cells were lysed using glass beads and centrifuged to remove cell debris, and bacteria were frozen in medium containing $20 \%$ fetal bovine serum (FBS, Invitrogen, Cergy Pontoise, France) and 5\% dimethylsulfoxide until use. The bacterial titer was determined by serial dilutions of bacterial preparations, and quantified using indirect 
immunofluorescence (see below). In some experiments, $O$. tsutsugamushi were killed by heating at $100^{\circ} \mathrm{C}$ for 5 minutes (HK bacteria).

Peripheral blood mononuclear cells were isolated from leukopacks (Etablissement Français du Sang, Marseille, France) over a Ficoll gradient (MSL, Eurobio, Les Ulis, France). Monocytes were isolated by adherence in 24-well plates with or without 12-mm glass coverslips and were differentiated into macrophages using a previously described procedure [19] with slight modifications. Briefly, monocytes were cultivated in RPMI 1640 containing 25 mM HEPES, 2 mM L-glutamine (Invitrogen) and 10\% heat-inactivated human AB serum (Lonza, Verviers, Belgium) for three days, and human serum was replaced by $10 \%$ FBS for additional periods ranging from an additional 4 to 6 days. Cultivated cells were designated as monocyte-derived macrophages as more than $90 \%$ of the cells did not express CD14 but did express CD68 as assessed by flow cytometry (Fig. S1). In some experiments, macrophages were treated with $50 \mathrm{ng} / \mathrm{ml}$ of recombinant human IFN- $\gamma$ (Peprotech France, Neuilly-sur-Seine) for 24 hours before infection, and IFN- $\gamma$ was added to cell preparations every day.

\subsection{Infection procedure}

Macrophages $\left(1.5 \times 10^{5}\right.$ cells per assay) were incubated in RPMI 1640 containing $25 \mathrm{mM}$ HEPES, 2 mM L-glutamine and 10\% heat-inactivated FBS in the presence of $O$. tsutsugamushi at a bacterium-to-cell ratio of 10:1 for 2 hours, a dose sufficient to determine bacterial uptake by monocytes [13]. Macrophages were washed to eliminate unbound bacteria and were cultivated for different time periods. The uptake and the intracellular fate of the bacterial pathogens was determined using confocal microscopy and quantitative real-time PCR (qPCR), as recently described [13]. For confocal microscopy, macrophages were infected for two hours, fixed with $3 \%$ paraformaldehyde, permeabilized with $0.1 \%$ Triton X100 and incubated with Alexa Fluor 488-phalloidin (Invitrogen) to stain filamentous actin and 
pooled serum from Thai patients with scrub typhus (1:400 dilution). Secondary antibodies consisted of goat anti-human IgG conjugated with Alexa Fluor 555 (Invitrogen).

Macrophages then mounted with Mowiol and were examined using a Leica TCS SP5 confocal microscope with a 63X/1.32-0.6 oil CS lens and an electronic 1.5X zoom. Images were processed with Adobe Photoshop V5.5 software. To isolate bacterial DNA, macrophages were lysed using $0.1 \%$ Triton X-100, and DNA was extracted in a $100 \mu \mathrm{l}$ volume using a QIAamp Tissue Kit (Qiagen, Courtaboeuf, France) and was stored at $-20^{\circ} \mathrm{C}$. qPCR was performed using $5 \mu$ of DNA extract, specific primers and probes and the Taqman system (Applied Biosystems, Warrington, UK). Primers and probe were constructed according to the available DNA sequence of O. tsutsugamushi strain Boryong (complete genome, GenBank ref. NC_009488.1). The forward primer consisted of 5'AAGCATAGGTTACAGCCTGGWGA-3'; the reverse primer consisted of 5'ACCCCAACGGATTTAATACTATATCWAC-3'; the probe consisted of 5'-FAMCCATCTTCAAGAAATGGCATATCTTCCTCAGG- TAMRA-3'. Each qPCR run included a standard curve generated from tenfold serial dilutions of a known concentration of bacterial DNA (Fig. S2), and a negative control consisted of DNA extracted from uninfected macrophages. The results are expressed as the total bacterial DNA copy number.

\subsection{Microarrays}

Macrophages $\left(4 \times 10^{5}\right.$ cells per assay) were incubated with $O$. tsutsugamushi at a bacteriumto-cell ratio of 10:1 for 8 hours. RNA was extracted from infected and uninfected macrophages using an RNeasy Mini kit (Qiagen) with a DNase digestion step. The quality of isolated RNAs was assessed using Agilent 2100 Bioanalyzer and an RNA 6000 Nano Kit (Agilent Technologies, Massy, France), and the quantity of RNAs was assessed using a NanoDrop 1000 spectrophotometer (Thermo Scientific, Wilmington, DE, USA). Eight RNA 
samples (four samples per experimental condition) were reverse transcripted in cDNA, and cDNA was labeled with Cy3 using a Low RNA Input Fluorescent Linear Amplification Kit (Agilent Technologies, Massy, France). The cDNA was hybridized to $4 \times 44 \mathrm{~K}$ microarray slides (Agilent Technologies). Scanned images were analyzed using Feature Extraction Software 10.5.1 (Agilent Technologies), and data processing and analyses were performed using Resolver 7.1 software (Rosetta Inpharmatics, Cambridge, MA), R software and BioConductor libraries, as described [13]. The Rosetta intensity error model for single color microarrays was used to normalize inter-array values. Statistical supervised analysis was performed using the significance analysis of microarrays, and the genes displaying an absolute fold change (FC) greater than 2.0 and a False Discovery Rate value less than 0.01 were considered to be differentially expressed. Results are expressed as FC calculated from the average of the four samples of unstimulated and $O$. tsutsugamushi-stimulated macrophages. The differentially expressed genes were classified on the basis of their Gene Ontology (GO) category as assessed using the DAVID bioinformatics tools [20]. To assess the M1/M2 polarization status of macrophages stimulated with $O$. tsutsugamushi, 30 genes known to be associated with M1 polarization and 22 M2 genes were manually selected from the literature [21-23]. Data were entered in the ArrayExpress database according to the Minimum Information About a Microarray Experiment procedure and can be accessed using the Gene Expression Omnibus series accession number at http://www.ncbi.nlm.nih.gov/geo/query/acc.cgi?acc=GSE38194.

\subsection{Quantitative real time $R T-P C R$}

Quantitative real time RT-PCR (qRT-PCR) was performed as described previously [13]. In brief, total RNA was isolated from macrophages using a Qiagen kit, and cDNA was synthesized using an oligo(dT) primer and M-MLV reverse transcriptase (Invitrogen). PCR 
was carried out using specific primers designed with primer3 software [24] (primers are the list in Table 1) and with Light Cycler-FastStart DNA MasterPLUS SYBR Green I master mix (Roche Applied Science, Meylan, France). We verified that the expression of the housekeeping gene encoding $\beta$-actin was not modulated in macrophages stimulated with $O$. tsutsugamushi, and we calculated the FC values in the expression of genes relative to the expression of $\beta$-actin gene using the $2^{-\Delta \Delta \mathrm{Ct}}$ method, where $\Delta \Delta \mathrm{Ct}=(\mathrm{CtTarget}-\mathrm{CtActin})_{\text {stimulated }}$ macrophages - (CtTarget - CtActin $)_{\text {unstimulated }}$ macrophages and Ct values were defined as the number of cycles for which the fluorescence signals were detected [19].

\subsection{TdT-mediated dUTP nick-end labeling (TUNEL) assay}

Infected macrophages were fixed with 3\% paraformaldehyde, and cell death was determined using In Situ Cell Death Detection Kit, TMR red (Roche Applied Science) as described previously [13]. 4',6-diamidino-2-phenylindole, dihydrochloride (DAPI) was used to counterstain nuclei. The number of TUNEL-positive cells and the number of DAPI-stained nuclei were determined using fluorescence microscopy. Cell death is expressed as the number of TUNEL-positive cells relative to the number of DAPI-stained nuclei.

\subsection{Immunoassays}

The levels of Tumor Necrosis Factor (TNF) and IFN- $\beta$ in macrophage supernatants were determined using TNF and IFN- $\beta$ ELISA kits, respectively (R\&D Systems, Lille, France). IL$1 \beta$ levels were measured using a specific ELISA kit provided by Diaclone (Besançon, France). The detection limit of the kits was $5 \mathrm{pg} / \mathrm{ml}$ for TNF, $10 \mathrm{pg} / \mathrm{ml}$ for IL- $1 \beta$, and $8 \mathrm{pg} / \mathrm{ml}$ for IFN- $\beta$. The intra- and interassay coefficients of variation of the ELISA kits ranged between 5 and $10 \%$. 


\subsection{Statistical analysis}

Results were compared using the non-parametric Mann-Whitney $U$ test and are expressed as means \pm standard deviation (SD). Differences were considered significant when $p$ values were less than 0.05 .

\section{Results}

\subsection{Intracellular fate of O. tsutsugamushi in macrophages}

The uptake of $O$. tsutsugamushi by macrophages was determined by confocal microscopy. Bacteria could be detected within macrophages after 30 minutes, and several bacteria were present in macrophages after two hours (Fig. 1A). The intracellular replication of $O$. tsutsugamushi was determined by incubating macrophages with organisms (10 bacteria per macrophage) for two hours and removing free bacteria by washing (considered to be day 0 ). Infected macrophages were cultured for an additional 5 days. At day 0 , the number of bacterial DNA copies was $6.9 \pm 1.5 \times 10^{4}$ and increased approximately twofold each subsequent day to reach $35 \pm 2 \times 10^{5}$ bacterial DNA copies after 5 days (Fig. 1B). Therefore, O. tsutsugamushi is able to replicate in macrophages.

\subsection{Global transcriptome analysis of $O$. tsutsugamushi-infected macrophages}

To understand how $O$. tsutsugamushi replicates within macrophages, we compared the transcriptional profile of macrophages stimulated with $O$. tsutsugamushi for 8 hours to that of unstimulated macrophages using whole genome microarrays. We found that 379 probes (364 upregulated and 15 downregulated probes) were modulated in response to O. tsutsugamushi infection. The hierarchical clustering computed from modulated genes revealed that $O$. tsutsugamushi-stimulated macrophages were markedly different from control macrophages (Fig. 1C). The annotation of the modulated genes was enriched for functions related to the 
immune response and host defense (e.g. immune response, chemotaxis, regulation of cytokine effector process, cytokines and inflammatory response), apoptosis and cell signaling (TLR signaling pathway) (Fig. 1D). These data demonstrate that O. tsutsugamushi stimulates the expression of specific genes that generate a transcriptional signature, including genes involved in the host response to infection.

\subsection{Analysis of the responses induced by $O$. tsutsugamushi}

From the macrophage genes that were differentially expressed in response to $O$. tsutsugamushi, we selected genes for further characterization belonging to the categories described above and that have been reported to be modulated in human monocytes stimulated with $O$. tsutsugamushi [13]. These genes included those associated with the type I IFN pathway, the inflammatory response and apoptosis.

The differential modulation of genes encoding IFN- $\beta$ and ISGs, such as OAS1 and MX1, which were upregulated in the microarray, was confirmed by qRT-PCR using the macrophages from three different donors stimulated with $O$. tsutsugamushi. The upregulated expression of these genes was long lasting, as the increase remained evident after 18 hours (Fig. 2A). This type I IFN response of $O$. tsutsugamushi-stimulated macrophages was also observed at the protein level. While unstimulated macrophages were unable to release IFN- $\beta$ (less than $8 \mathrm{pg} / \mathrm{ml}$ ), the amount of IFN- $\beta$ released by macrophages in response to $O$. tsutsugamushi was of $33 \pm 9$ and $40 \pm 3 \mathrm{pg} / \mathrm{ml}$ after 24 and 48 hours, respectively (Fig. 2E). We likewise selected genes related to inflammation that were upregulated in the microarray and analyzed them by qRT-PCR. We found that genes associated with chemotaxis (CCL5, CCL20, CXCL10 and CXCL11) were upregulated after 8 and 18 hours of stimulation with $O$. tsutsugamushi (Fig. 2B). Genes encoding inflammatory cytokines (TNF, IL-1ß, IL-16, IL- 
12p40, IL-23p19 and IL-15) were also upregulated; however this upregulation was transient

for the gene encoding IL-1 $\beta$ (Fig. 2C). It is possible that this robust modulation of inflammatory genes is associated with the M1 polarization of macrophages. To address this, we compared the transcriptional profile of $O$. tsutsugamushi-infected macrophages with 30 genes representative of M1 macrophages and 22 genes representative of M2 macrophages [21-23]. Of the M1-associated genes, 18 were upregulated (Table 2), while only 2 of the M2associated genes were upregulated following $O$. tsutsugamushi infection (Table 3). The majority of M2-associated genes were downregulated (Table 3), demonstrating that $O$. tsutsugamushi induces an M1-like transcriptional profile in macrophages. Similar to the type I IFN data, this change in the expression of inflammatory genes was reflected at the protein level. Unstimulated macrophages released about $40 \mathrm{pg} / \mathrm{ml}$ TNF and $20 \mathrm{pg} / \mathrm{ml} \mathrm{IL-1} \beta$ after 24 hours, but macrophages released high levels of TNF and IL-1 $\beta$ in response to bacterial infection (Fig. 2E).

We also evaluated the differential expression of genes associated with apoptosis, including TRAIL, XAF1 and AIM2 genes by qRT-PCR. We found that these genes were upregulated following 8 and 18 hours of stimulation with O. tsutsugamushi (Fig. 2D). Interestingly, $O$. tsutsugamushi induced apoptosis in 13\% and $18 \%$ of macrophages incubated with pathogens for 1 and 2 days, respectively, whereas only $1 \%$ of unstimulated macrophages and $3 \%$ of macrophages incubated with HK organisms were apoptotic (Fig. 3).

\subsection{Macrophage response to heat-killed O. tsutsugamushi}

To address the dependence of the macrophage response to $O$. tsutsugamushi on bacterial viability, we stimulated macrophages with heat-killed bacteria. In contrast to live $O$. tsutsugamushi, HK bacteria were unable to alter the type I IFN pathway; the expression of genes encoding IFN- $\beta$, OAS1 and MX1 was similar in macrophages stimulated with HK 
bacteria compared to unstimulated macrophages (Fig. 4A), and HK bacteria did not induce

IFN- $\beta$ release by macrophages (Fig. 4B). While genes associated with chemotaxis, such as CXCL1O and CXCL11, were not modulated in response to HK O. tsutsugamushi, genes encoding TNF, IL-6, IL-12p40 and IL-15 were upregulated (Fig. 4A). This response was however reduced compared to that induced by live organisms. Finally, the expression of IL$1 \beta$ and IL-23p19 genes was similar in macrophages stimulated with live and HK organisms (compare Fig. 4A and Fig. 2C). Additionally, HK O. tsutsugamushi induced a lesser release of TNF than live organisms and were unable to induce the release of IL-1 $\beta$ by macrophages (Fig. 4B). These results suggest that the type I IFN and, to a lesser degree, the response of macrophages to $O$. tsutsugamushi require live organisms.

\subsection{Effect of IFN- $\gamma$ on the intracellular fate of $O$. tsutsugamushi}

As O.tsutsugamushi polarized macrophages toward an M1 phenotype, we evaluated whether the M1 polarization of macrophages by IFN- $\gamma$ affected the intracellular fate of $O$. tsutsugamushi. On day 1 , the number of copies of bacterial DNA in IFN- $\gamma$-treated macrophages decreased compared to day 0. However, the number of copies of bacterial DNA moderately increased on day 2 and day 3 to reach the level observed at day 0 , and $O$. tsutsugamushi replicated within macrophages on days 4 and 5 (Fig. 5A). We expressed these results as the ratio of the number of copies of bacterial DNA in IFN- $\gamma$-treated macrophages to that of untreated macrophages. It was apparent that the treatment of macrophages with IFN- $\gamma$ prior to infection did not alter the uptake of bacteria. In contrast, the replication of $O$. tsutsugamushi was significantly $(p<0.05)$ decreased in IFN- $\gamma$-treated macrophages compared to untreated macrophages throughout the infection (Fig. 5B), but it is important to note that the pre-treatment of macrophages with IFN- $\gamma$ did not induce the complete elimination of $O$. 
tsutsugamushi. Together, these results suggest that the intracellular survival of $O$.

tsutsugamushi is associated with the polarization status of macrophages.

\section{Discussion}

We showed in this study that $O$. tsutsugamushi is able to replicate within human macrophages. These results are consistent with previous data demonstrating that $O$.

tsutsugamushi is present in tissue macrophages from autopsied patients with scrub typhus [8, 25]. The survival of $O$. tsutsugamushi within macrophages may contribute to infection in organs such as the liver, spleen and lymph nodes in patients with scrub typhus.

The mechanisms of $O$. tsutsugamushi internalization and survival in macrophages are unknown; however, they appear to differ in part from those in monocytes. For example, the uptake of $O$. tsutsugamushi by macrophages is lower than that observed in monocytes, but $O$. tsutsugamushi replicates to a higher level in macrophages than in monocytes [13]. The absence of lipopolysaccharide and peptidoglycan in the $O$. tsutsugamushi cell wall suggests that Toll-like receptors do not play a role in the uptake of $O$. tsutsugamushi [9]. Nevertheless, heparin sulfate proteoglycans [9] and nucleotide-binding oligomerization domain-containing protein (NOD) 1 [26] may be involved in the recognition of extracellular and cytosolic $O$. tsutsugamushi, respectively, by endothelial cells. It is supposed that the bacterial $56-\mathrm{kDa}$ type-specific antigen and surface antigen $\mathrm{C}(\mathrm{ScaC})$ are involved in cell invasion [9]. In nonprofessional phagocytes [27] and polymorphonuclear leukocytes [28], O. tsutsugamushi is localized to the cytosol. It is likely, therefore, that $O$. tsutsugamushi virulence is associated with escape from the phagosome in macrophages, potentially under the control of the bacterial genes encoding TlyC and a putative phospholipase D [9].

Microarray analysis of infected macrophages revealed that $O$. tsutsugamushi induced sustained changes in the transcriptional program of these cells, including the upregulation of 
type I IFN genes and several ISGs. This long-lasting transcriptional effect was confirmed by qRT-PCR and at the protein level as demonstrated by the release of IFN- $\beta$. The type I IFN response may be related to the cytosolic localization of $O$. tsutsugamushi. It is known that viruses [29] and cytosolic bacteria [30, 31] activate the type I IFN pathway. HK bacteria, which are degraded in phagolysosomes, were unable to modulate the type I IFN response and induce IFN- $\beta$ release by macrophages as was previously reported for monocytes [13]. Although the role of the type I IFN pathway in host defense is still ambiguous [32], it is known that type I IFNs activate immune cells (cytotoxic T cells and NK cells) that are critical for the clearance of rickettsial infections [33, 34], but these cytokines seem to be detrimental to the host when infected with Listeria monocytogenes [35] or Tropheryma whipplei [30]. Recently, it has been proposed that type I IFNs serve as probacterial function through the suppression of IL-1 produced by myeloid cells [36].

The microarray analysis also showed that $O$. tsutsugamushi induced the upregulation of inflammatory cytokines and chemokines. It has been demonstrated that mice susceptible to O. tsutsugamushi produce greater amounts of inflammatory cytokines and chemokines compared to resistant mice [18]. The ability of $O$. tsutsugamushi to induce the release of IL$1 \beta$ suggests that $O$. tsutsugamushi activates the inflammasome [37], and that this response requires bacterial viability, in contrast to the other components of the inflammatory response. To define the inflammatory response of macrophages to $O$. tsutsugamushi within the context of M1/M2 polarization, we compared the transcriptional program of $O$. tsutsugamushistimulated macrophages with genes characteristic of M1 and M2 polarization [21-23]. We found that $O$. tsutsugamushi induced essentially an M1-type program in macrophages, which differ slightly from that elicited by $O$. tsutsugamushi in monocytes [13]. The upregulation of genes encoding CCR7, IL-2 receptor $\alpha$ chain and IL-23p19 was more pronounced in macrophages than in monocytes, whereas the expression of IL-6 was strikingly higher in 
monocytes than in macrophages. The transcriptional program of infected macrophages resulted in a biological response, as shown by the release of TNF and IL-1 $\beta$ by macrophages stimulated with $O$. tsutsugamushi. The high levels of TNF and IL-1 $\beta$ release suggested a poor role for immunoregulatory cytokines such as IL-10. It has been previously shown that IL-10 may play a role in $O$. tsutsugamushi infection. Indeed, the preincubation of $\mathrm{J} 774$ macrophages with O. tsutsugamushi inhibited LPS-induced production of TNF through IL-10 [38]. We found that the expression of the gene encoding IL-10 was not modulated in response to $O$. tsutsugamushi as determined by microarray. We hypothesize that the imbalance between inflammatory and immunoregulatory cytokines is subtle. First, J774 macrophages incubated with O. tsutsugamushi for 24 hours released high levels of both TNF and IL-10 [38]. Second, in patients with scrub typhus, higher concentrations of IFN- $\gamma$ and IL-10 were found during the acute phase of the disease compared with the convalescent phase, but the concentrations of TNF and IL-1 $\beta$ were similar in acute and convalescent phases of the disease [17].

M1 polarization of macrophages, which is typically induced by IFN- $\gamma$ in the presence of bacteria, causes these cells to become microbicidal toward intracellular microorganisms $[19,39]$. We therefore evaluated whether the pre-treatment of macrophages with IFN- $\gamma$ affected $O$. tsutsugamushi survival. We found that treatment with IFN- $\gamma$ led to reduced $O$. tsutsugamushi replication without eradicating the bacteria. Thus, despite the detrimental effect of M1 polarization on $O$. tsutsugamushi replication, the bacteria nevertheless induced this M1 polarization in macrophages. However, it is likely that other elements of the host response to O. tsutsugamushi contribute to bacterial replication. These factors include type I IFNs, which have been shown to be critical for the survival of Mycobacterium tuberculosis [36]. In addition, apoptosis may favor the replication of $O$. tsutsugamushi. We observed that $O$. tsutsugamushi infection was associated with the up-regulation of apoptosis-related genes and with the induction of apoptosis as seen in the TUNEL assay. O. tsutsugamushi induced 
apoptosis in macrophages to a greater degree than in monocytes, and the replication of $O$. tsutsugamushi was higher in macrophages than in monocytes [13], suggesting that bacterial replication and apoptosis are associated.

\section{Conclusions}

We demonstrated that human macrophages are targeted in vitro by $O$. tsutsugamushi, reinforcing the idea that tissue macrophages may be infected during scrub typhus. In macrophages, $O$. tsutsugamushi stimulated a type I IFN response, likely due to its cytoplasmic localization and its induction of an M1-type response. This exacerbated inflammatory response may be related to the inflammatory complications observed in scrub typhus.

\section{References}

[1] Watt G, Parola P. Scrub typhus and tropical rickettsioses. Curr Opin Infect Dis 2003;16:429-36.

[2] Seong SY, Choi MS, Kim IS. Orientia tsutsugamushi infection: overview and immune responses. Microbes Infect 2001;3:11-21.

[3] Chattopadhyay S, Richards AL. Scrub typhus vaccines: past history and recent developments. Hum Vaccin 2007;3:73-80.

[4] Tamura A, Ohashi N, Urakami H, Miyamura S. Classification of Rickettsia tsutsugamushi in a new genus, Orientia gen. nov., as Orientia tsutsugamushi comb. nov. Int. J Syst Bacteriol $1995 ; 45: 589-91$.

[5] Cho NH, Kim HR, Lee JH, Kim SY, Kim J, Cha S et al. The Orientia tsutsugamushi genome reveals massive proliferation of conjugative type IV secretion system and host-cell interaction genes. Proc Natl Acad Sci U S A 2007;104:7981-6. 
[6] Nakayama K, Yamashita A, Kurokawa K, Morimoto T, Ogawa M, Fukuhara M et al. The revealed massive gene amplification during reductive genome evolution. DNA Res 2008;15:185-99.

[7] Cho NH, Seong SY, Huh MS, Kim NH, Choi MS, Kim IS. Induction of the gene encoding macrophage chemoattractant protein 1 by Orientia tsutsugamushi in human endothelial cells involves activation of transcription factor activator protein 1. Infect Immun 2002;70:4841-50. [8] Moron CG, Popov VL, Feng HM, Wear D, Walker DH. Identification of the target cells of Orientia tsutsugamushi in human cases of scrub typhus. Mod Pathol 2001;14:752-9. [9] Ge Y, Rikihisa Y. Subversion of host cell signaling by Orientia tsutsugamushi. Microbes Infect 2011;13:638-48.

[10] Nacy CA, Meltzer MS. Macrophages in resistance to rickettsial infection: macrophage activation in vitro for killing of Rickettsia tsutsugamushi. J Immunol 1979;123:2544-9.

[11] Fukuhara M, Fukazawa M, Tamura A, Nakamura T, Urakami H. Survival of two Orientia tsutsugamushi bacterial strains that infect mouse macrophages with varying degrees of virulence. Microb Pathog 2005;39:177-87.

[12] Nacy CA, Meltzer MS. Macrophages in resistance to rickettsial infections: protection against lethal Rickettsia tsutsugamushi infections by treatment of mice with macrophageactivating agents. J Leukoc Biol 1984;35:385-96.

[13] Tantibhedhyabgkul W, Prachason T, Waywa D, El Filali A, Ghigo E, Thongnoppakhun W et al. Orientia tsutsugamushi stimulates an original gene expression program in monocytes: relationship with gene expression in patients with scrub typhus. PLoS Neglect Trop Dis 2011;5:e1028. 
[14] Paris DH, Phetsouvanh R, Tanganuchitcharnchai A, Jones M, Jenjaroen K, Vongsouvath $\mathrm{M}$ et al. Orientia tsutsugamushi in human scrub typhus eschars shows tropism for dendritic cells and monocytes rather than endothelium. PLoS Negl Trop Dis 2012;6:e1466.

[15] Chierakul W, de Fost M, Suputtamongkol Y, Limpaiboon R, Dondorp A, White NJ et al. Differential expression of interferon- $\gamma$ and interferon- $\gamma$-inducing cytokines in Thai patients with scrub typhus or leptospirosis. Clin Immunol 2004;113:140-4.

[16] de Fost M, Chierakul W, Pimda K, Dondorp AM, White NJ, Van der Poll. T Activation of cytotoxic lymphocytes in patients with scrub typhus. Am J Trop Med Hyg 2005;72:465-7. [17] Kramme S, An le V, Khoa ND, Trin le V, Tannich E, Rybniker J et al. Orientia tsutsugamushi bacteremia and cytokine levels in Vietnamese scrub typhus patients. J Clin Microbiol 2009;47:586-9.

[18] Yun JH, Koh YS, Lee KH, Hyun JW, Choi YJ, Jang WJ et al. Chemokine and cytokine production in susceptible $\mathrm{C} 3 \mathrm{H} / \mathrm{HeN}$ mice and resistant $\mathrm{BALB} / \mathrm{c}$ mice during Orientia tsutsugamushi infection. Microbiol Immunol 2005;49:551-7.

[19] Benoit M, Ghigo E, Capo C, Raoult D, Mege JL. The uptake of apoptotic cells drives Coxiella burnetii replication and macrophage polarization: a model for $\mathrm{Q}$ fever endocarditis. PLoS Pathog 2008;4:e1000066.

[20] Dennis G, Sherman BT, Hosack DA, Yang J, Gao W, Lane HC et al. DAVID: database for annotation, visualization, and integrated discovery. Genome Biol 2003;4:P3.

[21] Martinez FO, Gordon S, Locati M, Mantovani A. Transcriptional profiling of the human monocyte-to-macrophage differentiation and polarization: new molecules and patterns of gene expression. J Immunol 2006;177:7303-11.

[22] Mosser DM, Edwards JP. Exploring the full spectrum of macrophage activation. Nat Rev Immunol 2008;8:958-69.

[23] Benoit M, Desnues B, Mege JL. Macrophage polarization in bacterial infections. J 
Immunol 2008;181:3733-9.

[24] Untergasser A, Nijveen H, Rao X, Bisseling T, Geurts R, Leunissen JA. Primer3 Plus, an enhanced web interface to Primer3. Nucleic Acids Res 2007;35:W71-4.

[25] Tseng BY, Yang HH, Liou JH, Chen LK, Hsu YH. Immunohistochemical study of scrub typhus: a report of two cases. Kaohsiung J Med Sci 2008;24:92-8.

[26] Byrd TF, Horwitz MA. Interferon gamma-activated human monocytes downregulate transferrin receptors and inhibit the intracellular multiplication of Legionella pneumophila by limiting the availability of iron. J Clin Invest 1989;83:1457-65.

[27] Chu H, Lee JH, Han SH, Kim SY, Cho NH, Kim IS et al. Exploitation of the endocytic pathway by Orientia tsutsugamushi in nonprofessional phagocytes. Infect Immun 2006;74:4246-53.

[28] Rikihisa Y, Ito S. Intracellular localization of Rickettsia tsutsugamushi in polymorphonuclear leukocytes. J Exp Med 1979;150:703-8.

[29] Levy DE, Marie IJ, Durbin JE. Induction and function of type I and III interferon in response to viral infection. Curr Opin Virol 2011;1:476-86.

[30] Al Moussawi K, Ghigo E, Kalinke U, Alexopoulou L, Mege JL, Desnues B. Type I interferon induction is detrimental during infection with the Whipple's disease bacterium, Tropheryma whipplei. PLoS Pathog 2010;6:e1000722.

[31] Lebreton A, Cossart P, Bierne H. Bacteria tune interferon responses by playing with chromatin. Virulence 2012;3:87-91.

[32] Decker T, Muller M, Stockinger S. The yin and yang of type I interferon activity in bacterial infection. Nat Rev Immunol 2005;5:675-87.

[33] Billings AN, Feng HM, Olano JP, Walker DH. Rickettsial infection in murine models activates an early anti-rickettsial effect mediated by NK cells and associated with production of gamma interferon. Am J Trop Med Hyg 2001;65:52-6. 
[34] Walker DH, Olano JP, Feng HM. Critical role of cytotoxic T lymphocytes in immune clearance of rickettsial infection. Infect Immun 2001;69:1841-6.

[35] Carrero JA, Calderon B, Unanue ER. Type I interferon sensitizes lymphocytes to apoptosis and reduces resistance to Listeria infection. J Exp Med 2004;200:535-40. [36] Mayer-Barber KD, Andrade BB, Barber DL, Hieny S, Feng CG, Caspar P et al. Innate and adaptive interferons suppress IL-1alpha and IL-1beta production by distinct pulmonary myeloid subsets during Mycobacterium tuberculosis infection. Immunity 2011;35:1023-34. [37] Mariathasan S, Monack DM. Inflammasome adaptors and sensors: intracellular regulators of infection and inflammation. Nat Rev Immunol 2007;7:31-40.

[38] Kim MJ, Kim MK, Kang JS. Orientia tsutsugamushi inhibits tumor necrosis factor $\alpha$ production by inducing interleukine 10 secretion in murine macrophages. Microb Pathog 2006;40:1-7.

[39] Boehm U, Klamp T, Groot M, Howard JC. Cellular responses to interferon-gamma. Annu Rev Immunol 1997;15:749-95. 


\section{Legends of Figures}

Figure 1. O. tsutsugamushi infection of macrophages and microarray analysis

A, Macrophages were incubated with $O$. tsutsugamushi (10 bacteria per cell) for 2 hours, permeabilized and incubated with Alexa Fluor 488-phalloidin and with antibodies specific for O. tsutsugamushi. Cells were examined by confocal microscopy, and a representative micrograph is shown with filamentous actin in green and bacteria in red. B, Macrophages that were incubated with $O$. tsutsugamushi for 2 hours were washed to remove free organisms and were cultured for an additional 5 days. The number of copies of bacterial DNA was determined by $\mathrm{qPCR}$. The results are the mean \pm SD of three different experiments. $\mathbf{C}$, Genes modulated in macrophages that were stimulated with $O$. tsutsugamushi for 8 hours are represented as a heatmap with probes in rows and samples in columns. Dendograms show the results of the hierarchical clustering of the samples (top) and genes (left). Gene expression levels are color-coded from blue to red. D, Genes modulated in response to O. tsutsugamushi were analyzed using DAVID functional analysis tools. The results are displayed as a pie graph representing the genes that were specifically modulated.

Figure 2. Effect of $O$. tsutsugamushi on the transcriptional response of macrophages A-D, Macrophages were stimulated with $O$. tsutsugamushi for 8 and 18 hours. RNA was extracted and qRT-PCR was performed on genes found to be regulated in the microarray analysis, including genes associated with type I IFN (A) and chemotaxis (B), genes encoding cytokines $(\mathrm{C})$ and genes associated with apoptosis and microbicidal responses (D). Results are expressed as the relative expression of each gene (stimulated vs. unstimulated conditions) and represent the mean \pm SD of 3 independent experiments performed in triplicate. $\mathbf{E}$, Macrophages were stimulated with $O$. tsutsugamushi for different time periods. Culture 
supernatants were analyzed for the presence of IFN- $\beta$, TNF and IL-1 $\beta$ using specific immunoassays. The results are expressed in $\mathrm{pg} / \mathrm{ml}$ and are presented as the mean $\pm \mathrm{SD}$ of three experiments performed in duplicate.

Figure 3. Effect of $O$. tsutsugamushi on macrophage apoptosis

Macrophages were stimulated with O. tsutsugamushi for 2 days, and cell apoptosis was determined by TUNEL assay. A, unstimulated macrophages. B, O. tsutsugamushi-stimulated macrophages.

Figure 4. Macrophage responses to HK O. tsutsugamushi

A, Macrophages were stimulated with HK O. tsutsugamushi for 8 hours. RNA was extracted and qRT-PCR was performed. The results are expressed as the relative expression of each gene (stimulated vs. unstimulated conditions) and represent the mean \pm SD of 3 independent experiments performed in triplicate. B, Macrophages were stimulated with $O$. tsutsugamushi for different time periods. Culture supernatants were analyzed for the presence of cytokines using immunoassays. The results are expressed in $\mathrm{pg} / \mathrm{ml}$ and are presented as the mean $\pm \mathrm{SD}$ of three experiments performed in duplicate.

Figure 5. Effect of IFN- $\gamma$ on the intracellular fate of O. tsutsugamushi

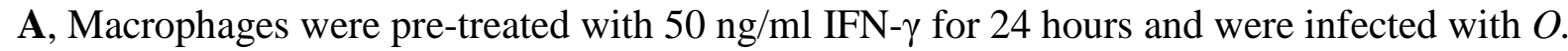
tsutsugamushi (bacterium-to-cell ratio of 10:1) for 2 hours. After washing, macrophages were cultured for 5 days in the presence of IFN- $\gamma$. The number of copies of bacterial DNA was determined by qPCR. The results are the mean \pm SD of three different experiments. $\mathbf{B}$, Macrophages pre-treated with IFN- $\gamma$ and control (untreated) macrophages were infected with O. tsutsugamushi as described above. The results are expressed as the ratio of the number of 
copies of bacterial DNA in IFN- $\gamma$-treated macrophages compared to control macrophages and the mean \pm SD of three different experiments. 


\section{Reviewer \#1}

1. p5, 2.1, line 6, the authors mentioned that they used CD14 and CD68 to differentiate monocytes from macrophages and there was more than $90 \%$ of the cells used were macrophages. It was not clear how this was determined, by flow cytometry? If so, can the author show the data after sorting as this is crucial for the experiment below?

The method (flow cytometry) to determine the percentage of macrophages is indicated in the new manuscript (page 5, lines 11-12). A representative experiment is also shown as supplementary data including how macrophages were collected (Figure S1).

2.p.5, line 8, IFN-gamma was maintained during the course of the experiment. Is there evidence to show that the recombinant IFN-gamma was stable for 24 hour at 1000U/ml under the culturing condition?

We previously showed that $1000 \mathrm{UI} / \mathrm{ml} \mathrm{IFN-gamma} \mathrm{was} \mathrm{effective} \mathrm{to} \mathrm{activate} \mathrm{macrophages}$ (ref 19). A concentration of $20 \mathrm{ng} / \mathrm{ml}$ IFN-gamma was recently used to activate human macrophages (Ouedraogo et al. J Proteomics 2012, in press): this concentration corresponded to $400 \mathrm{UI} / \mathrm{ml}$ IFN-gamma. To ensure that IFN-gamma was efficient, macrophages were pretreated with $1000 \mathrm{UI} / \mathrm{ml}$ IFN-gamma for 24 hours, infected for 2 hours, and incubated with $1000 \mathrm{UI} / \mathrm{ml} \mathrm{IFN-gamma} \mathrm{every} \mathrm{day.} \mathrm{As} \mathrm{the} \mathrm{relationship} \mathrm{between} \mathrm{IFN-gamma} \mathrm{activity} \mathrm{and}$ IFN-gamma doses is not clear, we replace $1000 \mathrm{UI} / \mathrm{ml}$ by $50 \mathrm{ng} / \mathrm{ml} \mathrm{IFN-gamma.} \mathrm{These} \mathrm{points}$ are indicated in 2.1. (page 5, lines 13-14) and the legend of figure 5.

3. p.6. A known concentration of bacterial DNA. How the concentration of bacteria (Orientia) DNA was determined? The qPCR data, including the standard curve, should be provided as a supplement figure.

In a preliminary experiment, Orientia was propagated in L929 cells, and bacteria were isolated after cell lysis. The bacterial preparation was divided into two parts: the first one was used to isolate bacterial DNA using a QIAamp Tissue Kit as described in the manuscript and the second part was used to determine the bacterial concentration in this preparation. For that purpose, serial dilutions of the bacterial preparation were deposited on glass coverslips (under a known volume), and the concentration of bacteria was determined by indirect immunofluorescence with pooled serum from patients with scrub typhus and secondary FITCantibodies. A very simple calculation (one specific DNA sequence per bacterium) allows the determination of bacterial DNA concentration per sample.

The qPCR data including the standard curve are now presented in 2.2. (page 6, lines 9-17) and as a supplemental figure (Figure S2).

4. The bacterium-to-cell ratio in 2.2 and 2.3 was different (10:1 to do infection and 20:1 to prepare samples for microarrays). Can the authors provide a rationale?

The bacterium-to-cell ratio in 2.3 was wrong. It was 10:1 in both 2.2 and 2.3., and corrections are included in the new manuscript (2.3. page 6, line 21).

5. p6, line 13. RNA was amplified. How RNA was amplified?

The sentence has been modified in 2.3. (page 7, line 1). 
6. p7. FC in the expression of genes relative to the expression of $\beta$-actin was computed. The basic assumption by the authors here was: The expression of $\beta$-actin gene is not altered upon infection with Orientia over the time course studied. Is there any evidence to support this assumption? It has been observed that the actins are involved in the cytoskeletal changes in rickettsia infected cells. It is possible that the expression level of $\beta$-actin might be changed. A better normalization standard is $18 S$ RNA gene.

We agree that the expression of b-actin may be altered by Orientia infection. We studied the expression of b-actin gene by unstimulated and Orientia-stimulated macrophages using microarray. We found no difference. We also tested the gene encoding GAPDH, another housekeeper gene. Its expression was similar in unstimulated and Orientia-stimulated macrophages, demonstrating that the genes encoding b-actin and GAPDH are relevant to quantify qRT-PCR data. This precision has been added in 2.4. (page 8, lines 3-5).

7. p.8, Figure 1 D. The criteria for the selection of modulated genes should be clearly stated. There were four samples per experiment condition (p6). What is the consistency in expression levels among the results from four quadruplet samples? Were the results in figure $1 C$ derived from the average of four sets of data or a representative of one sample?

We did not use four quadruplet samples, but four samples per experimental condition (unstimulated macrophages and Orientia-stimulated macrophages). The reproducibility of these samples was shown in Figure 1C, and the inter-array values were normalized using the Rosetta intensity error model for single color microarrays. These precisions have been added in 2.3. (page 7, lines 7-8).

The results in Figure 1D were derived from the average of the four sets of data. This information has been added in 2.3 (page 7, lines 11-13).

8. The regulation of $M 1$ genes and $M 2$ genes (Figure 3) should be presented as a table listing all those regulated genes.

The M1 genes and the M2 genes are presented in the new manuscript as Table 2 and Table 3, respectively.

9. In the results session, 3.3 on page 10 line \#14, it should be Figure $2 D$ rather than Figure $3 D$. Furthermore, the percentage of apoptotic cells were $12 \%$ and $17 \%$ in Orientia infected macrophages but only $2 \%$ in HK Orientia. What is the background level of apoptosis in macrophages without any stimulation? Or was the $12 \%$ already corrected for the background? Since the data is available, the reviewer would suggest showing the image of apoptotic cells as demonstrated by TUNEL assay.

Fig3D has been replaced by Fig2D.

The background level of apoptosis in unstimulated macrophages was about $1 \%$. The percentage of apoptotic cells is presented in the new section 3.3 (page 11, lines 17-19). The images of unstimulated and Orientia-stimulated macrophages are presented as a new Figure (Figure 3).

10. For Figure $2 E$ and $4 B$, it appeared that only the results of the IFN- $\beta, T N F$ and IL-1 $\beta$ from supernatant of Orientia infected and HK Orientia infected macrophage are shown. What about the unstimulated samples since the case was made that a significant increase was observed, it is important that the readers can see the background level (if any). In addition, 
IFN- $\beta$ is the only one that had two time points of 24 and $48 \mathrm{~h}$ while the other two had 8 and 24 time points. Can the authors provide a justification for the time difference?

Unstimulated macrophages did not release IFN-b (less than $8 \mathrm{pg} / \mathrm{ml}$ ). This information was in the new 3.3. (page 10, lines 19-20). They released low levels of TNF or IL-1b, and this information is given in the new paragraph 3.3. (page 11, lines 11-12). In addition, the detection limits and intra and interassay coefficients of variation of ELISA kits is given in the new paragraph 2.6 (page 8, lines 22-24).

In response to numerous pathogens, the inflammatory response occurs some hours after bacterial stimulation (justifying 8 and 24 hours) and decreased thereafter. The IFN response is delayed as compared to TNF response, and its maximum is comprised between 24 and 48 hours. The release of TNF and IL-1b of macrophages stimulated with Orientia was studied at 8 and 24 hours, as previously described for monocytes stimulated with Orientia (see ref 13). As the activation of IFN-b gene was sustained (upregulation at 8 and 24 hours), we tested the release of IFN-b not only at 24 hours (see ref 13) but also at 48 hours. We found no difference between 24 and 48 hours.

11. In Figure 5B, the "100\% IFN+/IFN-ratio was obtained after pretreating host cells with IFN (or not) for the same period of time, incubated with Orientia (a given number of what ratio?) followed by washing to remove unassociated Orientia then extract DNA from the infected cells to determine the \# of Orientia organisms? For Figure 5B, should the Y axis be specified $\times 10^{\wedge} 4$ or something similar as shown in Figure $1 B$ ? How the concentration of $1000 \mathrm{U} / \mathrm{ml}$ of IFN-?? was determined? Is it possible to carry out the experiment at a higher concentration to totally eradicate Orientia?

The bacterium-to-cell ratio (10:1) is now presented in the legend of figure 5 .

The precise value of bacterial DNA copies was lacking on the $\mathrm{Y}$ axis of figure 5A. This value is specified in the new figure $5 \mathrm{~A}$.

The concentration of IFN-gamma was highly effective. The concentration of $1000 \mathrm{UI} / \mathrm{ml}$ was higher than that used by Dellacasagrande et al. (J. Immunol. 2002,169:6309-15) and Ouedraogo et al. (J. Proteomics, 2012, in press). We also added IFN-gamma to macrophages every day whereas Benoit et al. (PLoS Pathog. 2008, 4:e1000066) added IFN-gamma every three days. The bacterial replication observed from day 4 to day 5 was not due to a lack of IFN-gamma because IFN-gamma was added to infected macrophages every day. This information has been added in 2.1. (page 5, line 14).

12. Many experiment details were referred to previous study (Ref 13). However, important information should still be included in this $M S$.

Experiment details are included in the new manuscript. See 2.1. (page 4, lines 21-24 and page 5, line 1); 2.2. (page 6, lines 9-17); 2.3. (page 6, lines 23-25; page 7, lines 3, 7-8 and 11-13); 2.4. (page 8 , lines 6-8); 2.5. (page 8 , lines 13-14); 2.6. (page 8 , lines 22-24). We also added two references (ref 22 and 23) to describe the selection of M1/M2 genes.

Discussion:

1. At the end of discussion, a comment was made about bacterial replication within host cells and apoptosis of host cells are associated. From day 4 to day 5, the increase in DNA copies was about 3 fold. Does this indicate the adaptation of Orientia in cultured macrophages? Can the authors speculated any survival advantage for this to occur in the macrophage at faster 
rate (both apoptosis and replication) or does it mean macrophages may not be a "natural" host cells for Orientia infection although in vitro it can be used as a host cell?

The number of bacterial DNA copies increased 3 fold from day 4 to day 5 in macrophages treated or not with IFN-gamma, suggesting the adaptation of Orientia to macrophages. This rate of replication, after a transient eclipse, was similar to that found in L929 cells but largely decreased compared to that observed in monocytes (see ref 13). On the other hand, Orientia induced a higher level of apoptosis in macrophages than in monocytes (compare our results with ref 13). The decreased bacterial replication and the increased apoptosis in macrophages compared with monocytes suggest that both events are associated.

The hypotheses of the reviewer are interesting. As macrophages seem to be a natural host for Orientia (see reference 8 for human infection and reference 10 for murine infection), we believed rather to an adaptation of Orientia to macrophages.

However, the underlying mechanisms and the biological signification of the interaction between macrophages and Orientia are unknown. As they are not under the scope of our paper, we prefer to avoid speculations.

\section{IL-10 secretion was observed in murine macrophages(J774, Kim MJ et al Microbial} Pathogenesis 2005) and in acute patient samples (Ref 17, 2009). Orientia inhibits TNF- $\alpha$ by inducing IL-10 secretion. It has been shown that TNF- $\alpha$ was associated with the scrub typhus diseases severity.

Why IL-10 was not selected for this study. Is it because the level of IL-10 was not changed in this study? At least the potential effect of IL-10 should be discussed.

We found that the gene encoding TNF and genes encoding inflammatory cytokines and chemokines were up-regulated in $\mathrm{O}$. tsutsugamushi-stimulated macrophages. The release of TNF was also dramatically increased in infected macrophages, suggesting a poor role for immunoregulatory cytokines such as IL-10. In addition, the expression of IL10 gene was not modulated in response to $\mathrm{O}$. tsutsugamushi as determined by microarray.

The potential role of IL-10 in O. tstsugamushi infection has been added in the new Discussion (4. page 15, lines 3-13) with a new reference (ref 38). 
Click here to download e-component: FigS1PDF.pdf e-component
Click here to d 
Click here to download e-component: FigS2PDF.pdf

e-component
Click here to d

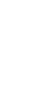

.

.

(

.

.

(

(

(

(

(

(

(

(

.

t

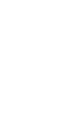

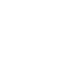

.

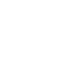

-
\end{abstract}

Clic

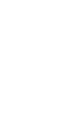

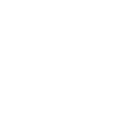

$\sqrt{2}$



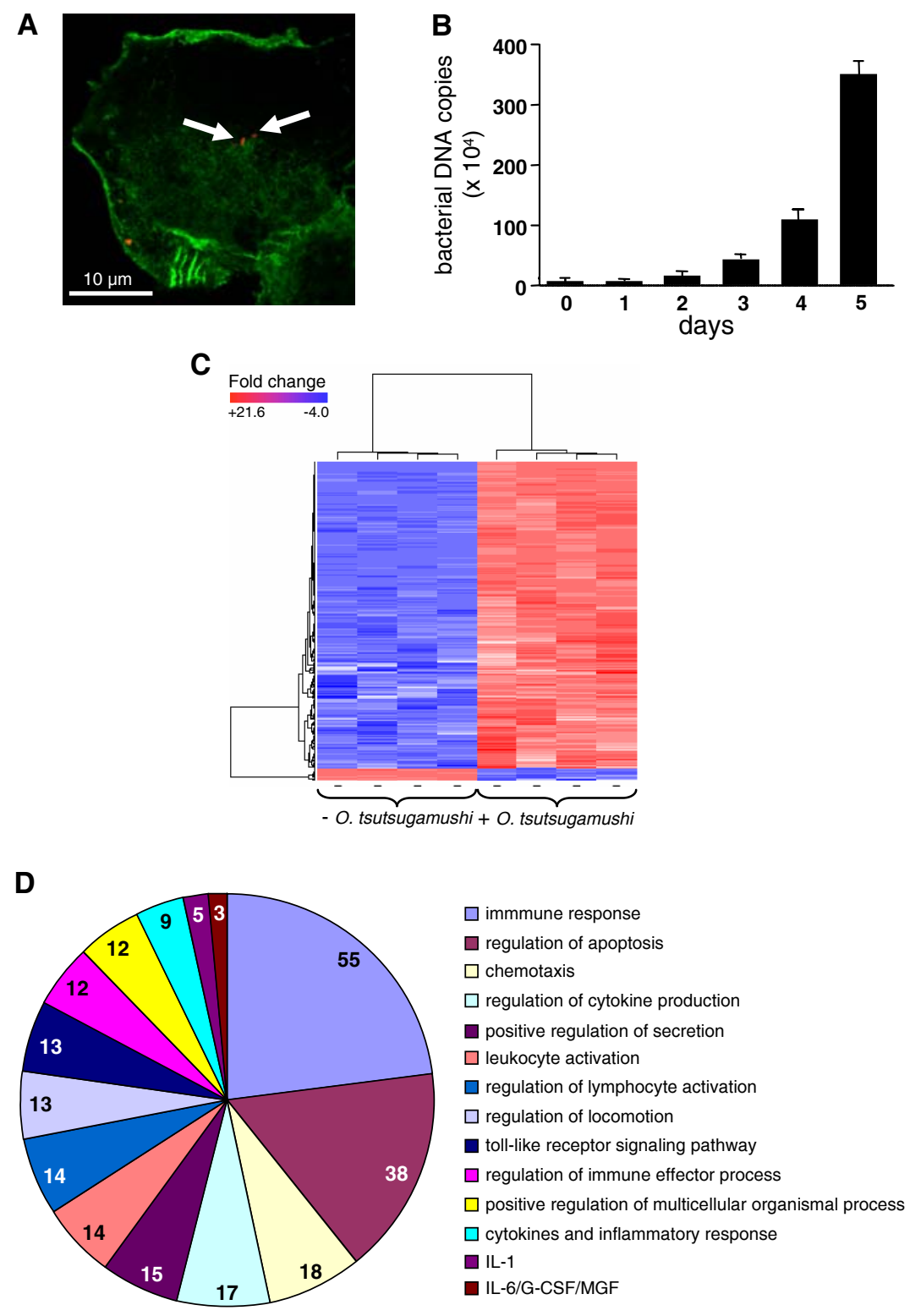

$\square$ immmune response

$\square$ regulation of apoptosis

$\square$ chemotaxis

$\square$ regulation of cytokine production

$\square$ positive regulation of secretion

$\square$ leukocyte activation

$\square$ regulation of lymphocyte activation

$\square$ regulation of locomotion

- toll-like receptor signaling pathway

$\square$ regulation of immune effector process

$\square$ positive regulation of multicellular organismal process

$\square$ cytokines and inflammatory response

$\square \mathrm{IL}-1$

IL-6/G-CSF/MGF

Figure 1 
A

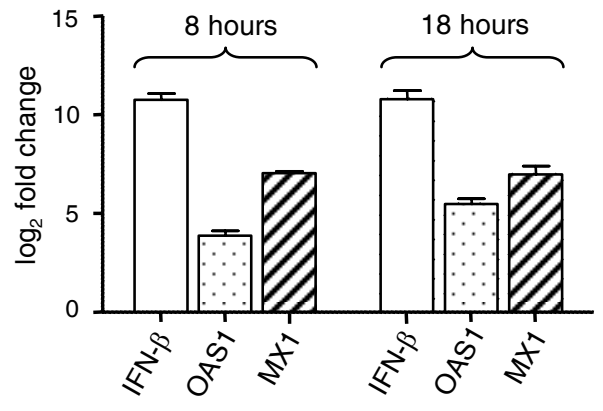

C

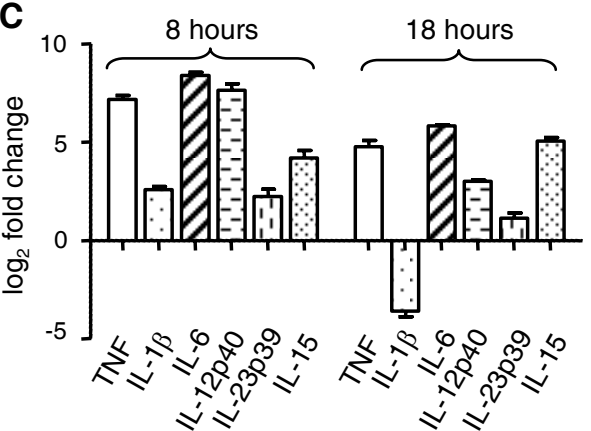

\section{B}
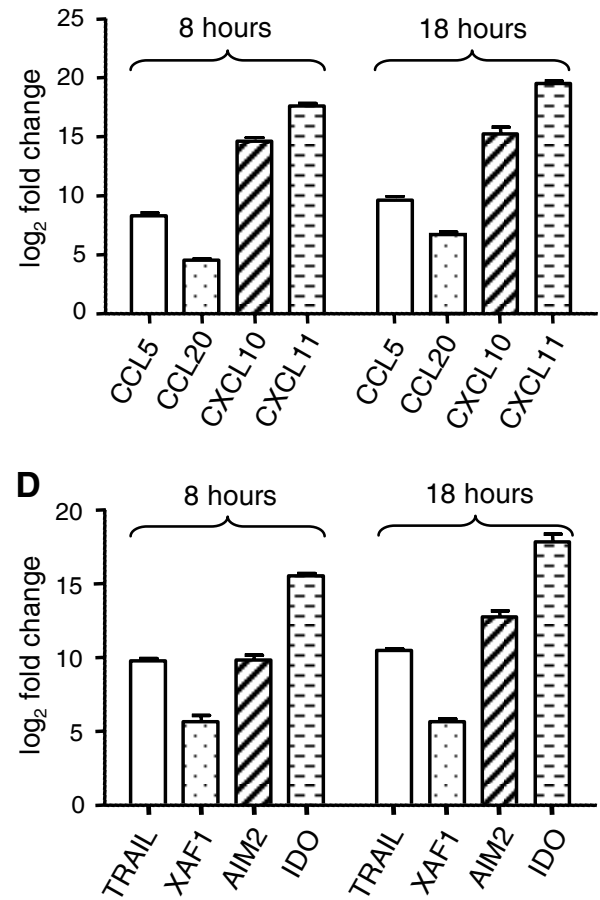

E

\begin{tabular}{|c|c|c|c|c|c|}
\hline \multicolumn{2}{|c|}{ IFN- $\beta(\mathrm{pg} / \mathrm{ml})$} & \multicolumn{2}{|c|}{ TNF (pg/ml) } & \multicolumn{2}{|c|}{$\mathrm{IL}-1 \beta(\mathrm{pg} / \mathrm{ml})$} \\
\hline $24 \mathrm{~h}$ & $48 \mathrm{~h}$ & $8 \mathrm{~h}$ & $24 \mathrm{~h}$ & $8 \mathrm{~h}$ & $24 \mathrm{~h}$ \\
\hline $33 \pm$ & $40 \pm 3$ & $7286 \pm 712$ & $3329 \pm 464$ & $533 \pm 11$ & $410 \pm 35$ \\
\hline
\end{tabular}

Figure 2 
A

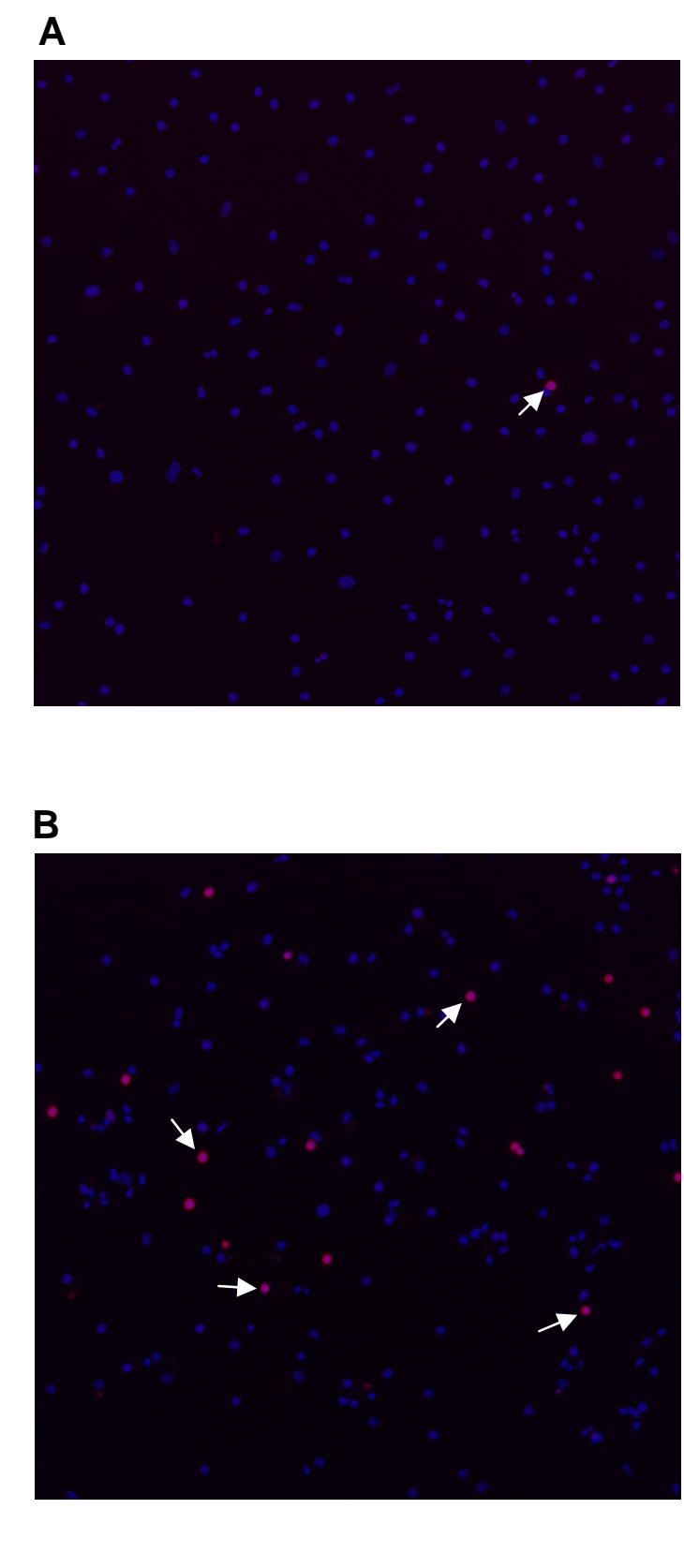

B

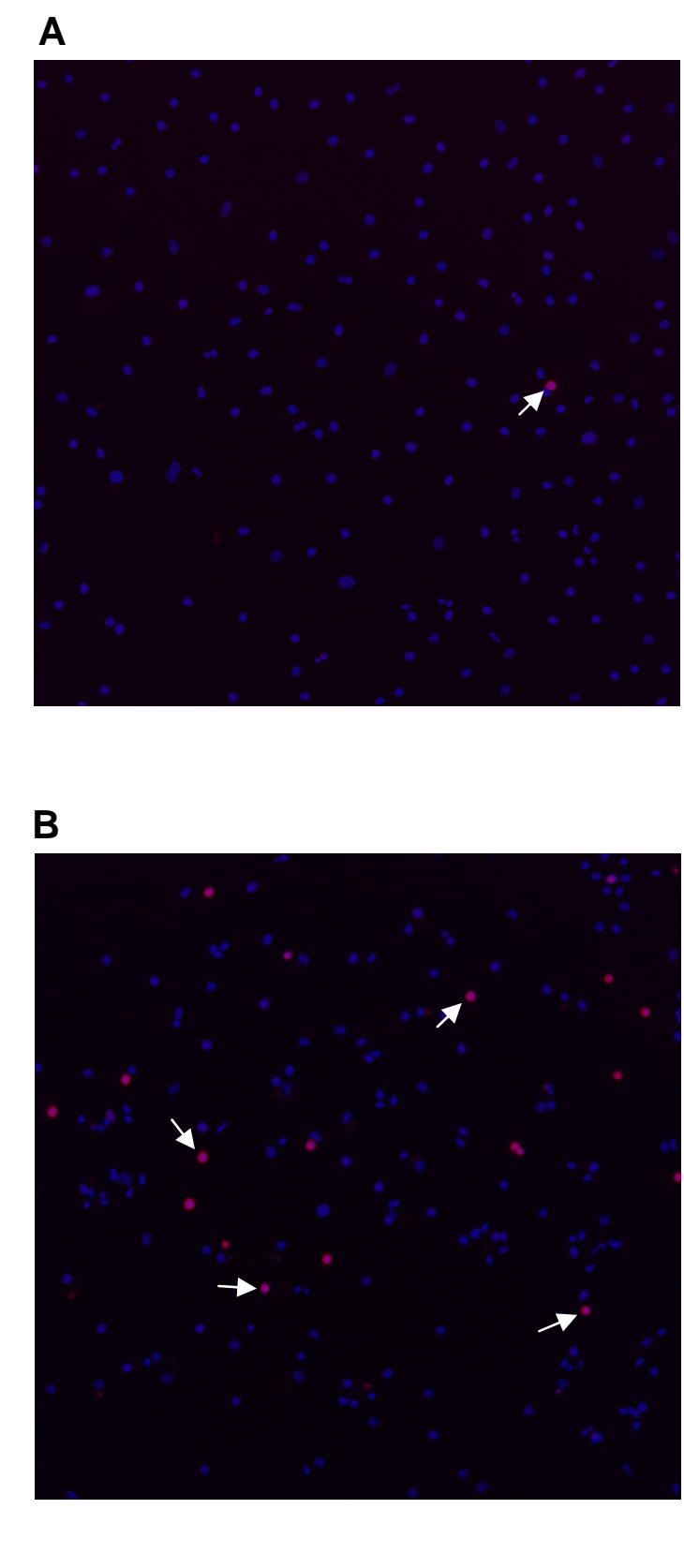

Figure
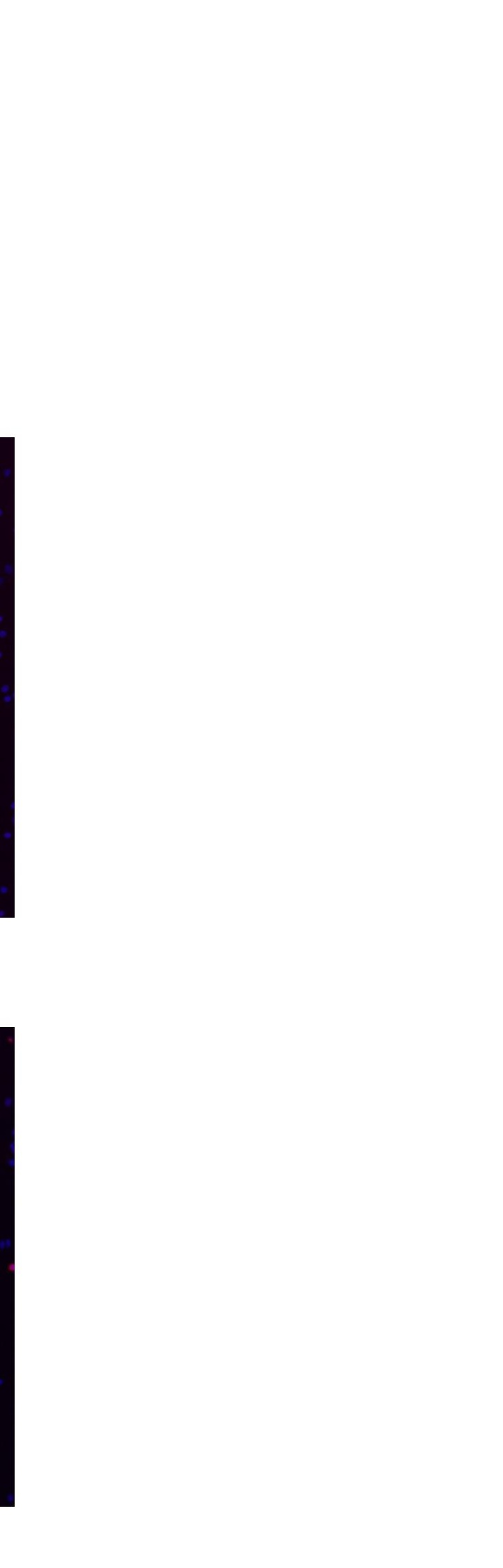


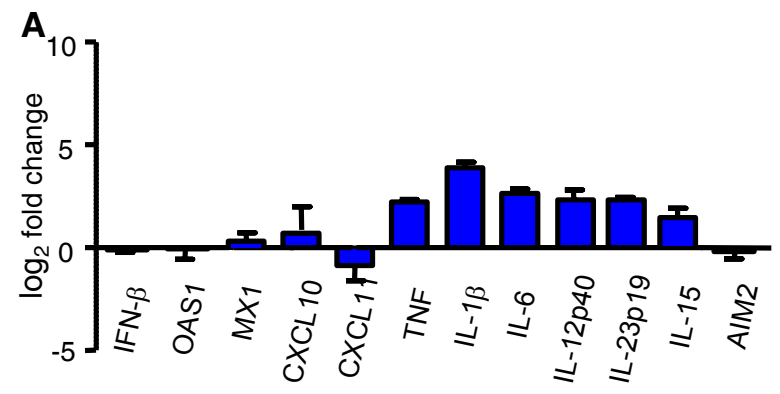

B

\begin{tabular}{|c|c|c|c|c|c|}
\hline \multicolumn{2}{|c|}{ IFN- $\beta$ (pg/ml) } & \multicolumn{2}{|c|}{ TNF (pg/ml) } & \multicolumn{2}{|c|}{$\mathrm{IL}-1 \beta(\mathrm{pg} / \mathrm{ml})$} \\
\hline $24 \mathrm{~h}$ & $48 \mathrm{~h}$ & $8 \mathrm{~h}$ & $24 \mathrm{~h}$ & $8 \mathrm{~h}$ & $24 \mathrm{~h}$ \\
\hline$<25$ & $<25$ & $911 \pm 102$ & $28 \pm 3$ & $<15$ & $<15$ \\
\hline
\end{tabular}

Figure 4 


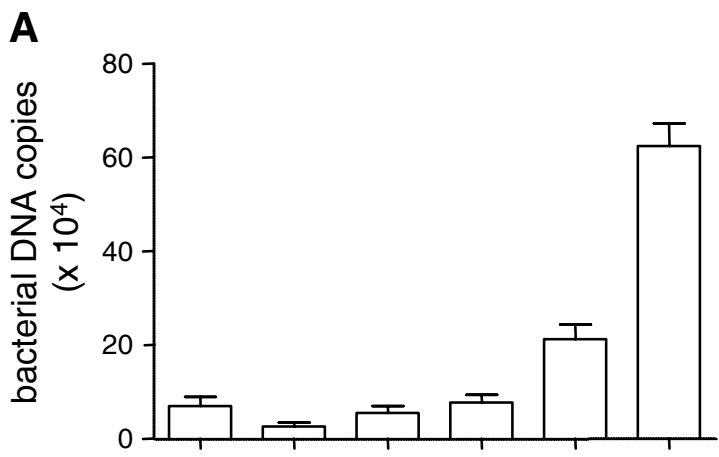

B

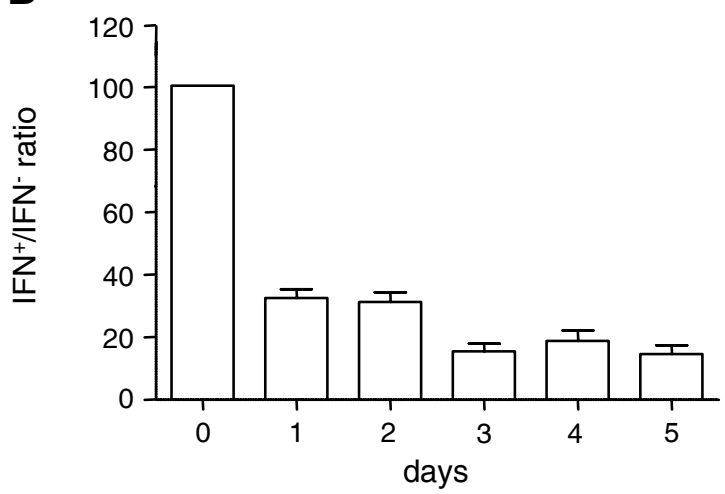

Figure 5 
Table 1. Nucleotide sequences of oligonucleotide primers

\begin{tabular}{lll}
\hline Symbol & $\mathbf{5}$ '-3' forward primers & 5'-3' reverse primers \\
\hline GAPDH & GGTGGTCTCCTCTGACTTCAACA & GTTGCTGTAGCCAAATTCGTTGT \\
AIM2 & GGTTTGTTTGTAGTCCAGAAGGTA & GTGCTATGAACTCCAGATGTCAG \\
CCL5 & CTGCTGCTTTGCCTACATTGC & GTTCAGGTTCAAGGACTCTCCATC \\
CCL20 & GACATCAATGCTATCATCTTTCAC & GCTATGTCCAATTCCATTCCA \\
CXCL10 & AAGCAGTTAGCAAGGAAAGGTC & TTGAAGCAGGGTCAGAACATC \\
CXCL11 & TATAGCCTTGGCTGTGATATTGTG & CTGCCACTTTCACTGCTTTTACC \\
IDO & TGCTGGTGGAGGACATGCTG & TGAAAGGACAAACTCACGGACTGA \\
IFN $\beta$ & GACGCCGCATTGACCATCTA & CCTTAGGATTTCCACTCTGACT \\
IL-1 3 & GCTGAGGAAGATGCTGGTTC & TCCATATCCTGTCCCTGGAG \\
IL-6 & TACCCCCAGGAGAAGATTCC & TTTTCTGCCAGTGCCTCTTT \\
IL-12p40 & AGGGGACAACAAGGAGTATGAGT & AGGGAGAAGTAGGAATGTGGAGT \\
IL-15 & GTTAGCAGATAGCCAGCCCATAC & TACTCAAAGCCACGGTAAATCC \\
IL-23p19 & AGGAGAAGAGGGAGATGAAGAGAC & GCTATCAGGGAGCAGAGAAGG \\
MX1 & GCCACCACAGAGGCTCTCAG & CTCAGCTGGTCCTGGATCTCCT \\
OAS1 & TCCGCCTAGTCAAGCACTGGTA & CCTGGGCTGTGTTGAAATGTGT \\
TRF & CCCGACTATCTCGACTTTGC & AGGTTGAGGGTGTCTGAAGGA \\
\hline XAF1 & GGCTAACTGACCTGGAAAGAAA & TTTGGTTGTGGCTGCTCTACT \\
\hline
\end{tabular}


Table 2. Modulation by O. tsutsugamushi of M1-related genes

\begin{tabular}{lllll}
\hline Gene Symbol & NCBI Gene ID & ENSEMBL Gene ID & PROBE_ID & FC \\
\hline CCR7 & 1236 & ENSG00000126353 & A_23_P343398 & 8.31 \\
IL2RA & 3559 & ENSG00000134460 & A_23_P127288 & 3.12 \\
IL7R & 3575 & ENSG00000168685 & A_23_P404494 & 1.76 \\
IL15RA & 3601 & ENSG00000134470 & A_23_P138680 & 2.70 \\
CCL2 & 6347 & ENSG00000108691 & A_23_P89431 & 1.31 \\
CCL3 & 6348 & ENSG00000006075 & A_23_P373017 & 4.54 \\
CCL4 & 6351 & ENSG00000129277 & A_23_P207564 & 2.52 \\
CCL5 & 6352 & ENSG00000161570 & A_23_P152838 & 1.94 \\
CCL15 & 6359 & ENSG00000161574 & A_24_P301501 & 1.22 \\
CCL19 & 6363 & ENSG00000172724 & A_23_P123853 & 1.86 \\
CCL20 & 6364 & ENSG00000115009 & A_23_P17065 & 2.01 \\
CXCL9 & 4283 & ENSG00000138755 & A_23_P18452 & 1.46 \\
CXCL10 & 3627 & ENSG00000169245 & A_24_P303091 & 3.49 \\
CXCL11 & 6373 & ENSG00000169248 & A_24_P20607 & 11.09 \\
CXCL16 & 58191 & ENSG00000161921 & A_23_P38505 & 1.17 \\
IL8 & 3576 & ENSG00000169429 & A_32_P87013 & 3.18 \\
TYMP & 1890 & ENSG00000025708 & A_23_P91802 & 1.13 \\
IL1A & 3552 & ENSG00000115008 & A_23_P72096 & 4.82 \\
IL1B & 3553 & ENSG00000125538 & A_23_P79518 & 5.92 \\
IL6 & 3569 & ENSG00000136244 & A_23_P71037 & 3.47 \\
IL12A & 3592 & ENSG00000168811 & A_23_P91943 & 1.31 \\
IL12B & 3593 & ENSG00000113302 & A_23_P7560 & 17.81 \\
IL23A & 51561 & ENSG00000110944 & A_23_P76078 & 5.12 \\
TNF & 7124 & ENSG00000232810 & A_24_P50759 & 3.03 \\
TNFSF10 & 8743 & ENSG00000121858 & A_23_P121253 & 5.23 \\
BCL2A1 & 597 & ENSG00000140379 & A_23_P152002 & 1.32 \\
FAS & 355 & ENSG00000026103 & A_23_P63896 & 2.05 \\
BIRC3 & 330 & ENSG00000023445 & A_23_P98350 & 1.43 \\
GADD45G & 10912 & ENSG00000130222 & A_24_P120934 & 1.25 \\
XAF1 & 54739 & ENSG00000132530 & A_23_P4283 & 2.64 \\
\hline
\end{tabular}

Macrophages were stimulated with $O$. tsutsugamushi for 8 hours, and microarrays were performed. M1-related genes $(n=30)$ were manually selected from the literature [2123]. The reference IDs of genes at NCBI and ENSEMBL are provided, as well as the probe ID on microarray. The ratio of gene expression of stimulated monocytes to control monocytes (FC) is shown. 
Table 3. Modulation by O. tsutsugamushi of M2-related genes

\begin{tabular}{lllll}
\hline Gene symbol & NCBI Gene ID & ENSEMBL Gene ID & PROBE_ID & FC \\
\hline CD36 & 948 & ENSG00000135218 & A_23_P111583 & 0.72 \\
CD163 & 9332 & ENSG00000177575 & A_23_P33723 & 0.75 \\
CD209 & 30835 & ENSG00000090659 & A_24_P186539 & 0.90 \\
CXCR4 & 7852 & ENSG00000121966 & A_23_P102000 & 1.42 \\
CD302 & 9936 & ENSG00000241399 & A_24_P396702 & 0.79 \\
CLEC7A & 64581 & ENSG00000172243 & A_23_P2640 & 0.73 \\
P2RY14 & 9934 & ENSG00000174944 & A_24_P165864 & 0.47 \\
HRH1 & 3269 & ENSG00000196639 & A_23_P212179 & 0.81 \\
MSR1 & 4481 & ENSG00000038945 & A_23_P216176 & 1.13 \\
MS4A4A & 51338 & ENSG00000110079 & A_23_P75769 & 0.67 \\
MS4A6A & 64231 & ENSG00000110077 & A_23_P203376 & 0.52 \\
LPAR6 & 10161 & ENSG00000139679 & A_23_P2705 & 0.68 \\
TGFBR2 & 7048 & ENSG00000163513 & A_23_P211957 & 0.47 \\
CCL1 & 6346 & ENSG00000108702 & A_23_P49759 & 2.79 \\
CCL13 & 6357 & ENSG00000181374 & A_23_P26965 & 0.60 \\
CCL17 & 6361 & ENSG00000102970 & A_23_P26325 & 0.91 \\
CCL18 & 6362 & ENSG00000006074 & A_23_P55270 & 1.11 \\
CCL22 & 6367 & ENSG00000102962 & A_24_P313418 & 0.95 \\
CCL23 & 6368 & ENSG00000167236 & A_24_P133905 & 1.03 \\
CCL24 & 6369 & ENSG00000106178 & A_23_P215491 & 1.05 \\
IL1RN & 3557 & ENSG00000136689 & A_32_P38125 & 3.05 \\
IL10 & 3586 & ENSG00000136634 & A_23_P126735 & 1.12 \\
\hline
\end{tabular}

Macrophages were stimulated with O. tsutsugamushi for 8 hours, and microarrays were performed. M2-related genes $(n=22)$ were manually selected from the literature [2123]. The reference IDs of genes at NCBI and ENSEMBL are provided, as well as the probe ID on microarray. The ratio of gene expression of stimulated monocytes to control monocytes (FC) is shown. 